auf konkrete Fälle, der Einzelfallbezug. Mir fehlte kriminaltaktisches Vorgehen, Ermittlung des Täters und Entscheidung, ob die Beweislage für eine Anklage ausreicht, das (hoffentlich) faire Streiten vor Gericht, um einen möglichst gerechten Schuldausgleich zu erwirken, der dem Opfer - zumindest zu einem gewissen Maß - Genugtuung verschafft. Nach etwa zweieinhalb Jahren Tätigkeit beim Ministerium in Sachen „Bekämpfung häuslicher Gewalt“ wurde bei der Staatsanwaltschaft durch Pensionierung das Sonderdezernat „häusliche Gewalt“ frei. Diese Gelegenheit konnte ich mir nicht entgehen lassen und kehrte zu „meiner“ Behörde zurück. Das Dezernat „häusliche Gewalt“ platzte mittlerweile - unter anderem als Ergebnis meiner eigenen Öffentlichkeits-, Fortbildungs- und Aufklärungsarbeit im Ministerium - aus allen Nähten, so dass ich in den ersten drei Jahren von der Arbeitsmasse fast erschlagen wurde. Die Gelegenheit, die hohen Standards für die staatsanwaltschaftliche Arbeit in Fällen häuslicher Gewalt, die ich im Ministerium mitentwickelt hatte, selbst in der Praxis umzusetzen, war aber so faszinierend, dass ich mich willig und (zumindest meist) klaglos auch mit Spätschichten und Wochenendarbeit in die Arbeit kniete. Mittlerweile bearbeiten drei Dezernent(inn)en Fälle häuslicher und sexualisierter Gewalt in unserer Behörde, ich wurde zwischenzeitlich zur Gruppenleiterin ernannt, und das Schönste: die Arbeit macht immer noch - jedenfalls meistens - Spaß.
Heute kann ich auf einen überhaupt nicht gradlinigen und zielstrebigen „Karriereweg“ zurückblicken, der mich aber mit einigen Ausreißern in die eine und die andere Richtung genau dahin geführt hat, wo ich nun das machen kann, was mir Freude macht, was mir Befriedigung verschafft und was ich gut kann. Die verschiedenen „Schlenker“ auf andere Gebiete sehe ich heute nicht als Zeitverschwendung, sondern im Gegenteil als wertvolle Gelegenheiten zum Erwerb von Schlüsselkompetenzen, die mir kein Jurastudium, kein Referendariat beigebracht hätten. Das Hineinschnuppern in soziale Tätigkeiten, die Beschäftigung mit fremden Sprachen und Kulturen, nicht zuletzt der (damals in der Vor-Computer-Ära noch sogenannte) „Schreibmaschinen“schein, den ich für den Abschluss am Dolmetscherinstitut benötigte, die Erziehung von zwei Kindern und das Jonglieren zwischen Verpflichtungen der Arbeit einerseits und der Familie andererseits haben mir Kompetenzen verschafft, von denen ich heute bei meiner Arbeit zehre und die auch vom Arbeitgeber anerkannt werden, was sich in der Ernennung als Gruppenleiterin zeigte.

Daher möchte ich alle jungen Kolleginnen motivieren, den Blick über den Tellerrand zu wagen, die Zeit, die dies kostet, als sinnvolle Investition zu verstehen und daran zu denken, dass es sich bei juristischen Sachverhalten um Lebenssachverhalte handelt, die korrekt zu verstehen der Mensch auch Lebenserfahrung, Offenheit und Vorurteilsfreiheit braucht.

\title{
Rechtsanwältin und Notarin
}

\section{Jutta Wagner}

Präsidentin des djb; Rechtsanwältin und Notarin, Berlin

Der Gedanke, Anwältin zu werden, war zwar während meiner Schulzeit einmal aufgetaucht, obwohl es in der Familie und im näheren Umfeld keine Vorbilder gab. Letztlich jedoch habe ich, wie so viele, 1969 das Jurastudium begonnen, nicht um einen bestimmten juristischen Beruf auszuüben, sondern um zwar auch diese Option zu haben, aber mindestens ebenso, um so oder so nützliche Kenntnisse auch für andere mögliche Laufbahnen zu erwerben.

Über mein Studium kann ich so gut wie nichts berichten, das in irgendeiner Art Vorbildcharakter haben und nachahmenswert sein könnte. Meine Studienjahre, zuerst in Hamburg, dann in Berlin, waren geprägt durch das Aufbegehren gegen überkommene, verkrustete Strukturen. Das hochschulpolitische Engagement und das allgemeinpolitische standen dabei nebeneinander.

Ich wurde zum studentischen Mitglied in einem Selbstverwaltungsgremium gewählt, in dem ich zum Beispiel gemeinsam mit Jutta Limbach an Konzepten zur Studienreform arbeitete. Gemeinsam mit zuerst nur drei anderen Kommilitonen baute ich am Fachbereich Rechtswissenschaft eine sehr schnell wachsende und erfolgreiche Studentengruppe, poli- tisch irgendwo zwischen SPD und Maoisten angesiedelt, auf. Die Organisation von großen Veranstaltungen, das Reden vor Hunderten von Menschen hat mich sicher etwas für das Leben, auch für das Anwältinnenleben, gelehrt, aber zum Erwerb von Rechtskenntnissen doch wenig beigetragen. Das gleiche gilt für die Demonstrationen und Veranstaltungen gegen den Vietnamkrieg.

Hinzu kam, dass ich mir meinen Lebensunterhalt durch fast durchgängige Teilzeitarbeit während des Studiums selbst verdienen musste.

Die Zeit fürs Studieren war also mehr als knapp und ich muss hier gestehen, dass ich an keiner einzigen Lehrveranstaltung kontinuierlich teilgenommen habe, außer an einem rechtshistorischen Seminar später nach dem Ersten Staatsexamen. Ich habe die notwendigen Klausuren und Hausarbeiten geschrieben, um möglichst schnell die vorgeschriebenen Scheine zu erwerben und mich in einer kleinen Arbeitsgruppe dann mit den Skripten von Alpmann-Schmidt auf das Erste Staatsexamen vorbereitet. Dazu habe ich mich dann nach der vorgeschriebenen Mindeststudienzeit, also nach sieben Semestern, angemeldet und es, wie sich leicht vorstellen lässt, mit mäßigem Erfolg bestanden. Aus heutiger Sicht ist es mir ein Rätsel, wie mir das überhaupt gelingen konnte und ich bin der festen Überzeugung, dass solches heute auch nicht mehr möglich wäre. 
Nach dem Ersten Staatsexamen habe ich das Angebot einer ziemlich linken und daher heute auch nicht mehr existenten Tageszeitung, für die ich nebenher immer geschrieben hatte, abgelehnt, dort in die Redaktion einzutreten. Ob diese Entscheidung mehr meiner wachsenden Skepsis gegenüber bestimmten politischen Heilsversprechen gedankt war oder meinem Bedürfnis, nicht auf halbem Wege stehen zu bleiben, weiß ich heute nicht mehr.

Jedenfalls waren es meine Ausbilderinnen und Ausbilder und die Ausbildung in der Referendarzeit, die mir erst die Freude daran nahe gebracht haben, als Juristin zu arbeiten. Für mich war es offensichtlich notwendig zu erleben, dass alles das praktische Relevanz hatte, was ich vorher häufig als reines Theoretisieren empfunden hatte.

Die Anwaltsstation am Ende des Referendardienstes habe ich nicht, wie viele andere, hauptsächlich als Vorbereitungsphase für das Zweite Staatsexamen betrachtet, sondern als Möglichkeit, möglichst viel über den Anwaltsberuf zu erfahren. $\mathrm{Zu}$ diesem Zweck habe ich mich ganz bewusst für einen Anwalt als Ausbilder entschieden, von dem bekannt war, dass seine Referendare weitgehend eigenständig arbeiten durften. Dieser Anwalt hat einen Beratungsvertrag mit einer kleinen Gewerkschaft, deren Mitglieder Anspruch auf Rechtsberatung hatten. Diese blieb weitgehend den Referendaren überlassen, sodass ich frühzeitig vom ersten Gespräch über die Beratung und Vertretung, die Klageerhebung bis zum Verhandlungstermin alles durchleben konnte, was das Anwaltsleben so ausmacht.

Von da an stand es für mich fest: Ich werde Anwältin. Dabei blieb ich auch, obwohl die im Vergleich zum Ersten Staatsexamen bessere Note im Zweiten Staatsexamen mir durchaus eine Bewerbung bei Justiz oder Verwaltung gestattet hätte.

Eine weitere Entscheidung, die ich kurzfristig nach dem Zweiten Staatsexamen treffen musste, war die über die Art meines anwaltlichen Berufsweges. Ich hatte das von vielen begehrte und attraktive Angebot, als Assistentin des Seniorpartners in die im damaligen Westberlin erfolgreichste und renommierteste wirtschaftsrechtliche Anwaltspraxis einzutreten.

Dieses Angebot habe ich nicht angenommen, sondern mich für einen viel schwierigeren Weg entschieden, den ich im Übrigen auch nicht zur Nachahmung empfehlen kann, auch wenn er in meinem Fall einen positiven Verlauf genommen hat.

Ich habe mich als selbständige Anwältin in Berlin in einer Bürogemeinschaft um die Ecke vom Kurfürstendamm niedergelassen. Den fixen Positionen auf der Ausgabenseite wie Raum-, Personal-, eigene Lebensunterhaltungskosten standen keinesfalls fixe Positionen auf der Einnahmenseite gegenüber.

Mein Schwerpunkt in der Referendarausbildung war das Arbeitsrecht. Nun musste ich feststellen, dass arbeitsrechtliche Mandate zu akquirieren gar nicht so einfach war. Ich hatte weder besondere Beziehungen irgendwelcher Art zu Arbeitnehmer-, noch zu Arbeitgeberorganisationen. Darüber hinaus sagte mir meine damalige Wahrnehmung jedenfalls ganz klar, dass zu jung und ohne Erfahrung und noch dazu weiblich zu sein, mindestens ein Negativkriterium zuviel war.
Ich machte also dieses und jenes, wie man das als Berufsanfänger, der nicht mit einem bestimmten Aufgabengebiet in eine größere Kanzlei einsteigt, ebenso macht. Es bildeten sich allerdings schnell zwei Schwerpunkte heraus, nämlich die Strafverteidigung und das Familienrecht.

Zur Strafverteidigung kam ich zunächst wegen meiner politischen Vergangenheit an der Universität. Es gab in Berlin damals eine sehr aktive Hausbesetzerbewegung, der wir, nebenbei gesagt, die Erhaltung ganzer historischer Stadtquartiere verdanken. Hausbesetzungen und Demonstrationen führten aber eben auch zu Strafverfahren und da mir Freisprüche gelangen, die etwas Aufsehen erregten, hatte ich Mandanten aus diesem Kreis. Dies zog andere strafrechtliche Mandate nach sich.

Familienrecht habe ich, wie damals üblich, an der Universität überhaupt nicht und während der Referendarausbildung auch nicht gelernt. Hinzu kommt, dass das Familienrecht sich gerade im Jahr vor meiner Zulassung zur Anwaltschaft, also 1977, geradezu revolutionär geändert hatte und ein völlig neues Feld für Anwaltschaft und Richter war. Hier wirkten sich, glaube ich, die zwei Negativkriterien jung und weiblich positiv aus und führten dazu, dass Menschen sich mit ihren Sorgen vertrauensvoll an mich wandten. Hier war es ein Erfolg, der gleichzeitig ein Misserfolg war, der mich in Bekanntheit und Renommee ein ganzes Stück voran brachte. Schon 1980, also als ganz junge Anwältin, habe ich eine Beschwerde zum Bundesverfassungsgericht erhoben, die vom ersten Senat zur Entscheidung angenommen wurde. Dies war um so ungewöhnlicher, als Berliner Bürger damals wegen des besonderen politischen Status von West-Berlin gar keine Verfassungsbeschwerden erheben durften und meine Mandanten daher erst von Berlin nach Hamburg umziehen mussten, um dies überhaupt zu ermöglichen. Entsprechend selten kamen daher auch Berliner Anwälte an das Bundesverfassungsgericht. Es ging um eine familienrechtliche Angelegenheit, ich habe damals erreichen wollen, dass auch nicht verheiratete Eltern die elterliche Sorge für ein gemeinsames Kind gemeinsam ausüben können. Dabei habe ich Mutter, Vater und Kind vertreten. Es wurde mit großem Aufsehen mündlich verhandelt, die Resonanz in den Medien war groß, aber letztlich hat damals eine knappe Mehrheit des Senats gegen mein Anliegen entschieden, eine Auffassung, die inzwischen längst revidiert ist.

Nach zwei etwas schwierigen Anfangsjahren konnte ich immerhin von meiner Arbeit einigermaßen leben und mich auf die Strafverteidigung und das Familienrecht konzentrieren. Einige Jahre später jedoch habe ich es mehr und mehr als Nachteil empfunden, mich auf zwei so verschiedenen Rechtsgebieten $\mathrm{zu}$ bewegen und mich entschlossen, die Strafverteidigung ganz aufzugeben zugunsten des Familienrechts.

Dies ist mir nicht leicht gefallen. Strafverteidigung ist etwas ganz Besonderes und hat mir viel Freude gemacht. Allerdings war, jedenfalls damals vor mehr als 20 Jahren, die Strafverteidigung fast eine reine Männerdomäne und es sind mir aus Berlin jedenfalls nur zwei Anwältinnen in Erinnerung, die sie einigermaßen erfolgreich ausgeübt haben. Seitdem betreibe 
ich im Bereich des Familien- und Erbrechts eine ständig wachsende Praxis.

1989 habe ich dann beschlossen, von der Möglichkeit Gebrauch zu machen, gleichzeitig Notarin zu werden. Grundsätzlich stehe ich allerdings dem Anwaltsnotariat kritisch gegenüber, aber das ist ein anderes Thema. Jedenfalls habe ich die nötigen Lehrgänge absolviert und Klausuren geschrieben und mit dem Erwerb dieses Titels, ohne dies so planen zu können, einen weiteren positiven Schritt in meiner beruflichen Entwicklung eingeleitet.

Gerade weil ich Notarin war, wurde ich 1997 gebeten, mich einer größeren überörtlichen Sozietät anzuschließen, die ihren Hauptsitz in Bonn hat und ein Büro in Berlin eröffnen wollte. Nach vielen Jahren als Einzelanwältin, wenn auch zeitweise in Bürogemeinschaft, fand ich dies außerordentlich reizvoll, habe mich dafür entschieden und dies bis heute nicht bereut. Es ist für mich außerordentlich interessant, engen Kontakt zu haben mit Kolleginnen und Kollegen, deren Berufsalltag so ganz anders ist als der meine. Sie sind überwiegend im öffentlichen Recht tätig und begleiten große Projekte, von denen man ständig in den Zeitungen lesen kann. Dies gilt im Grunde auch für die etwas kleinere, aber ebenfalls sehr erfolgreiche zivilrechtliche Abteilung.
Eine große Rolle in meinem anwaltlichen Berufsleben hat immer die ehrenamtliche Tätigkeit gespielt. Ich bin Mitglied des Berliner und damit des Deutschen Anwaltvereins geworden und wurde schon als relativ junge Anwältin 1985 in den Vorstand der Rechtsanwaltskammer Berlin gewählt. Für 12 Jahre war ich Richterin am Berliner Anwaltsgericht, zuletzt als Vorsitzende. Beides hat zwar viel Arbeit gemacht, ich habe dabei aber auch sehr, sehr viel gelernt darüber, wie andere arbeiten, welche Fehler häufig gemacht werden, wo es zu Konflikten mit anderen Anwälten, Gericht oder Mandanten kommt und vieles andere mehr. Acht Jahre lang war ich im Vorstand des Berliner Landesverbandes des djb, davon sechs Jahre als Vorsitzende und jetzt bin ich seit fast fünf Jahren Präsidentin des djb. Wenn im September 2011 meine Amtszeit ausgelaufen sein wird, lasse ich mir sicherlich wieder etwas Neues einfallen, das ich ehrenamtlich tun kann. Ob dies aber wieder und weiter etwas im juristischen Bereich sein wird, weiß ich noch nicht.

Ist dieses alles nun eine berufliche Entwicklung, die weder typisch männlich noch typisch weiblich ist? Gibt es typische Unterschiede und wenn ja, wo liegen sie? Meine „gefühlte“ Antwort und die Statistik sagen hier dasselbe: Meine berufliche Entwicklung ist in bestimmter Weise typisch für Anwältinnen.

\section{Syndikus-Anwältin}

\section{Dr. Thordis Koeppel}

Mitglied im Arbeitsstab Berufsorientierung und Karriereplanung des djb, Vorstandsmitglied im Landesverband Hamburg im djb von 2000 bis 2002; Justiziarin und Rechtsanwältin, Hamburg

Vor Kurzem fiel mir unsere Abitur-Zeitung in die Hände und ich habe mal wieder gelesen, was ich damals über meine Pläne geschrieben habe: „Zunächst will ich nach Frankreich reisen. Dann will ich ein Jurastudium irgendwo in Deutschland beginnen. Ich träume davon, in der weiten Welt herumzukommen. Wenn alle Stricke reißen, heirate ich halt."

Kurze Bilanz: Ich war nach dem Abitur zu einem Sprachkurs in Bordeaux. Mein Jurastudium habe ich im Herbst 1989 in Freiburg begonnen. In der weiten Welt bin ich ein bisschen herumgekommen und geheiratet habe ich im Jahre 2005. Aber nun der Reihe nach.

Studieren wollte ich eigentlich schon immer. Dies lag wohl vorrangig daran, dass mein Vater stets von seiner Studienzeit geschwärmt hat. Außerdem hat er immer propagiert, zum Studieren müsse man in eine andere Stadt gehen. Zuhause wohnen und studieren, das hätte meine Mutter vielleicht ganz gerne gesehen, aber dank der Einstellung meines Vaters habe ich mich nach dem Abitur an Unis in ganz Deutschland beworben und dass es Freiburg wurde (weiter konnte ich von Hamburg fast nicht wegziehen) lag zum einen daran, dass die Freiburger Universität mir als erste einen Studienplatz ange- boten hatte und zweitens daran, dass ich im Alter von 13 bereits einmal für eine Woche bei einer kanadischen Freundin unserer Familie im Studentenwohnheim in Freiburg übernachten durfte, während meine Eltern „bloß“ in einem Hotel wohnten.

Soweit ich mich erinnern kann, habe ich mich entschieden, Jura zu studieren, da ich in einem Prospekt des Auswärtigen Amtes gelesen hatte, dass für den Höheren Dienst überproportional viele Jurist(inn)en eingestellt würden. Ich hatte, wie vermutlich viele, den Traum, nach dem Studium in den Diplomatischen Dienst zu gehen. Auf diese Weise hätte ich mein Faible für Fremdsprachen und Reisen gut mit meinem Beruf verbinden können. Während meines Jurastudiums habe ich deshalb auch regelmäßig diverse Sprachkurse besucht und angefangen, Spanisch zu lernen.

So etwa im 3. Semester bin ich aufgrund eines Zeitungsartikels auf den djb aufmerksam geworden und habe mich über diesen informiert. Kurz darauf erhielt ich eine Einladung zum Stammtisch in Freiburg. Dort traf ich auf meine Strafrechts-AG-Leiterin, die mir auch schon vom djb erzählt hatte, und ehe ich mich versah, wurde ich aktives Mitglied im djb. Fortan habe ich die djb-Stammtische bis zum Ende meines Studiums mit organisiert und sicherlich Einiges dazu gelernt, was ich im Studium nicht gelernt hätte. Insbesondere den Kontakt zu bereits berufstätigen Juristinnen fand ich immer sehr spannend. 